\title{
Associations among Household Animal Ownership, Infrastructure, and Hygiene Characteristics with Source Attribution of Household Fecal Contamination in Peri-Urban Communities of Iquitos, Peru
}

\begin{abstract}
Francesca Schiaffino, ${ }^{1,2}$ Dixner Rengifo Trigoso, ${ }^{3}$ Josh M. Colston, ${ }^{4}$ Maribel Paredes Olortegui, ${ }^{3}$ Wagner V. Shapiama Lopez, Paul F. Garcia Bardales, ${ }^{3}$ Nora Pisanic, ${ }^{5}$ Meghan F. Davis, ${ }^{5,6}$ Pablo Penataro Yori, ${ }^{3,4}$ and Margaret N. Kosek ${ }^{3,4 *}$

${ }^{1}$ Department of International Health, Johns Hopkins Bloomberg School of Public Health, Baltimore, Maryland; ${ }^{2}$ Faculty of Veterinary Medicine, Universidad Peruana Cayetano Heredia, Lima, Peru; ${ }^{3}$ Asociacion Benefica Prisma, Iquitos, Peru; ${ }^{4}$ Division of Infectious Diseases and International Health, University of Virginia School of Medicine, Charlottesville, Virginia; ${ }^{5}$ Department of Environmental Health and Engineering, Johns Hopkins Bloomberg School of Public Health, Baltimore, Maryland; ${ }^{6}$ Department of Molecular and Comparative Pathobiology, Johns Hopkins Bloomberg School of Medicine, Baltimore Maryland
\end{abstract}

\begin{abstract}
Using previously validated microbial source tracking markers, we detected and quantified fecal contamination from avian species and avian exposure, dogs, and humans on household cooking tables and floors. The association among contamination, infrastructure, and socioeconomic covariates was assessed using simple and multiple ordinal logistic regressions. The presence of Campylobacter spp. in surface samples was linked to avian markers. Using molecular methods, animal feces were detected in $75.0 \%$ and human feces in $20.2 \%$ of 104 households. Floors were more contaminated than tables as detected by the avian marker Av4143, dog marker Bactcan, and human marker Bachum. Wood tables were consistently more contaminated than non-wood surfaces, specifically with the mitochondrial avian markers ND5 and CytB, fecal marker Av4143, and canine marker Bactcan. Final multivariable models with socioeconomic and infrastructure characteristics included as covariates indicate that detection of avian feces and avian exposure was associated with the presence of chickens, maternal age, and length of tenancy, whereas detection of human markers was associated with unimproved water source. Detection of Campylobacter in surface samples was associated with the avian fecal marker Av4143. We highlight the critical need to detect and measure the burden of animal fecal waste when evaluating household water, hygiene, and sanitation interventions, and the possibility of decreasing risk of exposure through the modification of surfaces to permit more effective household disinfection practices. Animals may be a more important source of household fecal contamination than humans in many low-resource settings, although interventions have historically focused almost exclusively on managing human waste.
\end{abstract}

\section{INTRODUCTION}

Domestic animal husbandry at the household level is a common practice in low-resource rural communities, contributing to intra-domiciliary fecal contamination and potentially increasing the risk of transmission of zoonotic enteric pathogens. ${ }^{1}$ Water, soil, and household surfaces are regularly contaminated with bacterial pathogens of both human and other animal fecal material. ${ }^{2-5}$ Detection and quantification of fecal contamination within the house is generally carried out using standard microbiologic methods targeting traditional fecal indicator bacteria (FIB), such as Escherichia coli. Fecal indicator bacteria are commonly detected and quantified in household water and surfaces, and epidemiologic studies have associated the degree of fecal contamination with hygiene, sanitation infrastructure, and sociodemographic characteristics of household members without accounting for the production of FIB from domestic and peri-domestic animal sources. ${ }^{4,6,7}$ One of the principal assumptions underpinning these studies is that fecal contamination is mainly human derived. However, animal fecal matter is often also highly prevalent within households, and the presence of animals, as well as animal fecal waste, has been associated with increased risk of enteric illness. ${ }^{8-10}$ Although traditional FIB are able to determine the degree of fecal contamination by taking into consideration all sources, these methods are not capable

\footnotetext{
${ }^{*}$ Address correspondence to Margaret N. Kosek, Division of Infectious Diseases, International Health, and Public Health Sciences, University of Virginia, 345 Crispell Dr., Rm. 2525, Charlottesville, VA
} 22903. E-mail: mkosek@virginia.edu of distinguishing between animal and human sources of detected feces, and this fundamentally stifles directed control measures. ${ }^{11,12}$ Furthermore, samples analyzed using traditional microbiologic methods are easily contaminated by FIB from other environmental sources or reservoirs, such as soils. ${ }^{13,14}$

Microbial source tracking (MST) methods have been developed to determine the source of fecal contamination within wastewater and recreational water systems for remediation purposes. ${ }^{15,16}$ By attributing and quantifying fecal contamination to a specific animal species, intervention strategies and remediation measures can be more easily targeted and applied. Several MST markers have been developed and standardized in rural communities where there is a high degree of household fecal contamination. ${ }^{17-21}$ However, to date and to our knowledge, no studies have applied MST methods to quantify animal fecal burden in household surfaces. Furthermore, few studies that have applied MST markers in low-income settings have included avian-specific markers of fecal contamination. ${ }^{22-24}$ This is of critical importance, given the role of poultry within domestic animal husbandry practices as an alternative source of income and nutritional source of protein, as well as a source of Campylobacter spp., one of the main causes of bacterial diarrhea, stunting, and environmental enteropathy in pediatric populations of low-income settings. ${ }^{25-28}$

Using MST markers previously validated in the same region, this study quantified the burden of animal fecal contamination of surfaces by animal species, in households in a peri-urban, low-resource, tropical community of Loreto, Peru, with a particular emphasis on avian fecal contamination. ${ }^{29}$ We assessed the associations between MST presence and 
burden in household surfaces with household infrastructure and socioeconomic characteristics of the primary caregiver. Finally, we assess the association between the detection of Campylobacter spp. and different MST markers in surface samples from the same households.

\section{MATERIALS AND METHODS}

Study setting and population. This study was carried out in Santa Clara de Nanay, Santo Tomas, and La Union $\left(3^{\circ} 47^{\prime} \mathrm{S}\right.$, $73^{\circ} 20^{\prime} \mathrm{W}$ ), three peri-urban communities located $15 \mathrm{~km}$ away from lquitos city center, Loreto, Peru. These communities combined are composed of approximately 1,300 households and 10,000 individuals. Common occupations for men in these communities include small-scale agricultural production, fishing, and moto-taxi driving, whereas women most commonly report being homemakers, having a small corner shop ("bodega") or being unemployed. ${ }^{30}$

Data and sample collection. Between October 2018 and September 2019, households from these three communities were randomly selected for sampling. Within each household, two surface samples were obtained, and a socioeconomic questionnaire was completed by the head of the household. Surfaces sampled included the main table where cooking took place and entrance floors, for reasons described previously. ${ }^{4}$ The cooking surface of preference (where food is manipulated for human consumption) was identified by the head of the household. As described previously, a $30.0-\mathrm{cm}$ by $30.0-\mathrm{cm}$ square of scrap paper used to frame the sampling area was placed on top of the selected surface. ${ }^{6,31}$ Using sterile nitrile gloves and applying moderate pressure, half of a dry autoclaved electrostatic cloth $\left(\right.$ Swiffer ${ }^{\circledR}$, Procter \& Gamble, Cincinnati, $\mathrm{OH}$ ) was spread over the framed surface area. The cloth was then placed in a sterile 24-oz Whirl-Pak bag (Nasco, $\mathrm{WI}$ ) and $10 \mathrm{~mL}$ of phosphate-buffered solution (PBS) added. Samples were placed in a cooler with ice-packs and transported to the laboratory within 4-6 hours of collection for processing.

If there was a child younger than 2 years present in the household, a plastic toy was given and exchanged for an identical item within 24 hours as a sentinel object that reports more directly on the microbial exposure of the mobile child. ${ }^{32}$ The toy was placed in a plastic bag, and $10 \mathrm{~mL}$ of PBS were added. All samples went through the same processing protocol. This sampling strategy was inspired by the work of Vujcic et al. ${ }^{32}$ in Bangladesh.

Sample processing. Samples were vigorously shaken for 5 minutes to ensure the sampled material was homogenized within the PBS solution. The solution was transferred into sterile $2 \mathrm{~mL}$ crioviales for DNA extraction. DNA was extracted from $500 \mathrm{~mL}$ of solution using PowerSoil ${ }^{\circledR}$ DNA extraction kit (Qiagen, Germantown, MD) following bead-beating according to the manufacturer's instructions. For each extraction, a negative control consisting of RNA-free water was used.

Quantitative polymerase chain reaction using microbial source tracking markers. Eight MST markers that have previously been validated within this context were used to score surface samples. ${ }^{29}$ Specifically, two avian fecal markers (Av4143 and LA35), two avian mitochondrial fecal markers (cytb and ND5), two human fecal markers (BachHum and HF83-Taqman), one dog fecal marker (BactCan), and one pig fecal marker (Pig2Bac) were used. ${ }^{33-37}$ Details regarding the target species, gene, and internal validity parameters of all eight MST markers are presented in Supplemental Table 1.

TaqMan assays consisted of final reaction mixtures of $20 \mu \mathrm{L}$, which included TaqMan ${ }^{\mathrm{TM}}$ Advanced Fast Start Master Mix $(\times 2)$ (Applied Biosystems, Foster City, CA), forward and reverse primers $(200 \mu \mathrm{M})$, probes $(100 \mu \mathrm{M}), 5 \mu \mathrm{L}$ of DNA template, and RNA-free water (Ambion ${ }^{\mathrm{TM}}$, Thermo Fisher Scientific, Waltham, MA). Negative controls consisting of RNA- and DNA-free water were included in each amplification reaction. Reaction mixtures were placed in a 96-well plate and amplified using a StepOnePlus real-time PCR system (Applied Biosystems). Internal amplification controls (qHsaCtIP0001003, Bio-Rad Laboratories, Irvine, CA) were run for every marker and surface sample, and runs were invalid if the internal standard did not amplify. Standard amplification conditions $\left(95^{\circ} \mathrm{C}\right.$ for 5 minutes, 40 cycles of $95^{\circ} \mathrm{C}$ for 15 seconds, $53^{\circ} \mathrm{C}$ for 15 seconds, and $60^{\circ} \mathrm{C}$ for 45 seconds) were used for all reactions, except for $L A 35$ and Av4143, for which annealing temperatures were set at $56^{\circ} \mathrm{C}$ and $55^{\circ} \mathrm{C}$, respectively. Standard curve analysis was performed as reported previously. ${ }^{29}$

Detection of Campylobacter spp. Surface samples were tested for the presence or absence of Campylobacter spp. using a semi-quantitative PCR that targeted a 16S sRNA segment that identifies all members of the Campylobacter genus $^{38}$ as well as the cadF gene (adhesion to fibronectin) to detect thermotolerant species (most likely Campylobacter jejuni/Campylobacter coli only ${ }^{39}$ ) (Supplemental Table 2). Final reaction mixtures of $25 \mu \mathrm{L}$ consisted of $12.5 \mu \mathrm{L}$ of Taq Environmental Master Mix 2.0 (Thermo Fisher Scientific), primers at a concentration of $0.2 \mu \mathrm{M}$ each, and the probes at a concentration of $0.1 \mu \mathrm{M}$ each, $1 \mu \mathrm{L}$ of DNA, and nuclease-free water. The assay was performed under the following cycling conditions: $95^{\circ} \mathrm{C}$ for 10 minutes, 45 cycles of $95^{\circ} \mathrm{C}$ for 15 seconds, and $55^{\circ} \mathrm{C}$ for 1 minute (StepOne Instrument, Applied Biosystems). A target was determined to be positive if a cycle threshold (Ct) of less than 38 was obtained for the $16 \mathrm{~S}$ gene.

Data analysis. A binary variable indicating the presence and absence of a given MST marker in a surface sample was created using the assay-specific limit of detection, indicating a positive sample if the Ct obtained was below the limit of detection (lower Ct) and a negative sample if the Ct was above that limit or was undetermined. Determination of assayspecific limits of detection has been described previously. ${ }^{29}$ Floor and table surface samples were treated as distinct independent samples. Floor was categorized as either finished-made of a material that separated the dirt floor from household members or animals-or unfinished-uncovered earth. ${ }^{40}$ The surface material of tables was classified as being either of wood or non-wood, the latter category mainly composed of wood tables covered with plastic sheeting. Pearson's chi-square and Fisher's exact test were used to test the differences in positivity for any specific MST marker between floors and tables, as well as between unfinished and finished floors, and wood and non-wood tables. Gene quantities were $\log _{10}$ transformed $\left(\log _{10}\right.$ gene copy number $\left.[\mathrm{GCN}] / \mu \mathrm{L}\right)$ ). The distribution of this continuous variable was assessed using Shapiro-Wilk, skewness, and kurtosis normality tests. Data were found to be right skewed for all six markers. The difference in the median $\log _{10} \mathrm{GCN} / \mu \mathrm{L}$ in tables and floors, as well as unfinished versus finished floors and non-wood versus wood floors was assessed using the Wilcoxon rank-sum test. Gene quantities in floor and table samples were independently 
categorized into tertiles and modeled as an ordinal outcome variable, where the first tertile was interpreted as "low," the second as "medium," and the third one as "high" GCN quantities.

Covariates analyzed included age of the primary caregiver (years), maternal education (years), age of the primary caregiver at first pregnancy (years), and average monthly income (US dollars). Household infrastructure characteristics included a binary variable for household crowding (less than six people living in the household/more than six people living in the household), length of household tenancy (less than 1 year, between 1 and 5 years, between 5 and 10 years, between 10 and 20 years, and more than 20 years), floor material (unfinished/finished), table material (wood/non-wood), and wall type (cement/other). Hygiene covariates included treatment of drinking water, water source (improved/unimproved), and sanitation facility (improved/unimproved), as defined by the WHO/UNICEF Joint Monitoring Program for Water Supply and Sanitation. ${ }^{41}$ Finally, the presence and absence of chickens within the household at the time of visit were also included as a covariate.

Baseline associations among the main exposures, floor material, and table material were performed using Pearson's chi-square or Fisher's exact test for binary covariates and Wilcoxon rank-sum test for continuous covariates. Regression models were fitted for all MST markers separately using simple and multiple ordered logistic regressions to test the association between the degree of contamination ("low," "medium," or "high") in floor and table samples, and the specified household and socioeconomic covariates. Multivariable regression models were fitted with all household and socioeconomic covariates. The proportional odds assumption was tested for all adjusted and unadjusted regression models.

Associations between MST marker detection and the presence of Campylobacter in floor and table samples were assessed by logistic regression with the presence of Campylobacter as the binary outcome variable and using the odds ratio $(\mathrm{OR})$ as the measure of effect. Separate models were run for each MST marker, and the presence of chickens in the household was included as a covariate in all models. Type I error was set at 0.05 for all statistical analyses. Data management and statistical analysis were performed in STATA 14 and (Stata Corp., CollegeStation, TX) and R (version 3.3.2, R Foundation for Statistical Computing, Vienna, Austria).

Ethical considerations. Ethical approval was obtained from the international review boards of Asociacion Benefica Prisma and Johns Hopkins Bloomberg School of Public Health. A local field-worker from Asociacion Benefica Prisma explained the study procedures to the household head, and signed informed consent was requested before any study procedure was initiated.

\section{RESULTS}

A total of 104 samples obtained from surfaces used for food preparation and 104 matched floor samples were obtained from 104 households. Of the 104 floor samples, 54 (51.9\%) were made of dirt, 39 (37.5\%) of cement, six (5.8\%) of wood, four $(3.8 \%)$ of tile, and one (1.0\%) floor was covered in plastic material. A total of 54 floors were classified as unfinished (i.e., bare earth), and 50 were classified as finished. Of the 104 table samples, $75(72.1 \%)$ were made of wood, $22(21.2 \%)$ of plastic, four (3.8\%) of fabric, two (1.9\%) of tile, and one $(1.0 \%)$ was covered in paper (Table 1). At least $76.9 \%(80 / 104)$ of households were positive for any fecal marker (Av4143, Bactcan, BacHum, and HF183-Taqman) in either floor or table samples. Animal feces were detected in $75 \%(78 / 104)$ of households, and human feces were detected in $20.2 \%$ (21/ 104) of households. Bivariate results of each MST marker by table and floor surface categories are shown in Table 2, quantitative results are shown in Figure 1, and categorical (high, medium, and low data) results for each MST marker are shown in Supplemental Table 3. All samples were negative for the avian fecal marker LA35 and pig fecal marker Pig2Bac. Covariate characteristics of household and primary caregiver are presented in Table 3. The univariate associations between MST gene quantity tertiles of floors and tables surface samples, and the household and primary caregiver covariates are shown in the Supplemental Material (Supplemental Tables 4 and 5), whereas the adjusted associations are presented in Table 4.

Microbial source tracking markers in surface samples. Avian fecal marker Av4143. Of the 104 floor samples, 49.0\% $(n=51)$ of floors and $15.4 \%(n=16)$ of tables $(P$-value $<0.001)$ were positive for the avian marker Av4143. The median $\log _{10}$ $\mathrm{GCN} / \mu \mathrm{L}$ of Av4143 was 1.80 among floor samples and 1.33 among table samples $(P$-value $<0.001)$. The number of finished and unfinished floors, as well as wooden and nonwooden tables positive for Av4143, was not significantly different. Similarly, the median $\log _{10} \mathrm{GCN} / \mu \mathrm{L}$ of Av4143 among finished and unfinished floors, and wood and nonwood tables was not statistically different. Fifty percent of floor samples and $16.3 \%$ of table samples were classified as having a "high" quantity of Av4143 marker, whereas $17.3 \%$ of floors and $12.5 \%$ of table samples were "medium," and $31.5 \%$ of floors and $71.2 \%$ of tables were "low" ( $P$-value $<0.001)$. The OR of a floor sample being classified in the "high" tertile in comparison with the "middle" and low" tertiles was 3.70 (95\% Cl: 1.71-7.99; $P$-value $=0.001$ ) among households who owned chickens in comparison to those who did not. This same statistically significant association was found among table samples scored for Av4143 (OR: 4.42; 95\% Cl: $1.70-11.49 ; P$-value $=0.003$ ). The OR of a table sample being classified in the "high" group in comparison to the "medium" and "low" groups was $3.32(95 \% \mathrm{Cl}$ : $1.05-10.32 ; P$-value = 0.041 ) among surfaces made of wood in comparison to material other than wood.

The multivariable model showed that the presence of chickens in the household (OR: 4.03; 95\% Cl: 1.51-10.79; $P$-value $=0.005)$, maternal age (OR: 0.93; 95\% Cl: 0.87-0.99;

TABLE 1

Materials of floor and table surface samples from households $(N=104)$ in three communities of lquitos, Loreto, Peru

\begin{tabular}{lcc}
\hline & \multicolumn{2}{c}{ Surfaces $(n=208)$} \\
\cline { 2 - 3 } Material & Floors $(n=104), n(\%)$ & Tables $(n=104), n(\%)$ \\
\hline Cement & $39(37.5)$ & $0(0.0)$ \\
Tile & $4(3.8)$ & $2(1.9)$ \\
Plastic & $1(9.6)$ & $22(21.2)$ \\
Dirt & $54(51.9)$ & $0(0.0)$ \\
Wood & $6(5.8)$ & $75(72.1)$ \\
Paper & $0(0.0)$ & $1(9.6)$ \\
Cloth & $0(0.0)$ & $4(3.8)$ \\
\hline
\end{tabular}


TABLE 2

Percent positive (A) floor and table samples, (B) unfinished and finished floors, and (C) wood and non-wood samples from households in three communities of lquitos, Peru, analyzed using MST markers

\begin{tabular}{|c|c|c|c|c|}
\hline & & Floors & Tables & \\
\hline MST & Target species & Percent positive $(n / M)$ & Percent positive $(n / M)$ & $P$-value* \\
\hline Av4143 & Domestic birds and waterfowl & $49.03(51 / 104)$ & $15.38(16 / 104)$ & $<0.001$ \\
\hline CytB & Chickens and ducks & $59.62(62 / 104)$ & $67.31(70 / 104)$ & 0.249 \\
\hline ND5 & Chickens and ducks & $83.65(87 / 104)$ & $77.88(81 / 104)$ & 0.291 \\
\hline Bactcan & Dogs & $27.88(29 / 104)$ & $11.54(12 / 104)$ & 0.003 \\
\hline Bachum & Humans & $17.31(18 / 104)$ & $3.85(4 / 104)$ & 0.003 \\
\hline \multirow[t]{2}{*}{ HF-183 Taqman } & Humans & $0.96(1 / 104)$ & $0.96(1 / 104)$ & NA \\
\hline & & Unfinished floors & Finished floors & \\
\hline MST marker & Target species & Percent positive $(n / M)$ & Percent positive $(n / M)$ & $P$-value* \\
\hline Av4143 & Domestic birds and waterfowl & $57.4(31 / 54)$ & $40.0(20 / 50)$ & 0.082 \\
\hline CytB & Chickens and ducks & $64.8(35 / 54)$ & $54.0(27 / 50)$ & 0.319 \\
\hline ND5 & Chickens and ducks & $88.9(48 / 54)$ & $78.0(39 / 50)$ & 0.185 \\
\hline Bactcan & Dogs & $35.2(19 / 54)$ & $20.0(10 / 50)$ & 0.125 \\
\hline Bachum & Humans & $15.0(8 / 54)$ & $20.0(10 / 50)$ & 0.606 \\
\hline \multirow[t]{2}{*}{ HF-183 Taqman } & Humans & $0.00(0 / 54)$ & $2.0(1 / 50)$ & 0.481 \\
\hline & & Wood tables & Non-wood tables & \\
\hline MST marker & Target species & Percent positive $(n / M)$ & Percent positive $(n / M)$ & $P$-value* \\
\hline Av4143 & Domestic birds and waterfowl & $18.7(14 / 75)$ & $7.0(2 / 29)$ & 0.224 \\
\hline CytB & Chickens and ducks & $72.0(54 / 75)$ & $55.2(16 / 29)$ & 0.101 \\
\hline ND5 & Chickens and ducks & $84(63 / 75)$ & $62.1(18 / 29)$ & 0.016 \\
\hline Bactcan & Dogs & $12.0(9 / 75)$ & $10.3(3 / 29)$ & 1.000 \\
\hline Bachum & Humans & $5.3(4 / 75)$ & $0.0(0 / 29)$ & 0.574 \\
\hline HF-183 Taqman & Humans & $1.3(1 / 75)$ & $0.0(0 / 29)$ & 1.000 \\
\hline
\end{tabular}

MST = microbial source tracking. A greater number of floor samples are positive for the presence of avian, dog, and human feces, and a greater number of wood tables are positive for avian mitochondrial DNA in comparison to non-wood tables. Bold values indicate $P$-value $<0.05$.

$P$-value $=0.044$ ), and length of property tenancy (more than 20 years in comparison to less than 1 year, OR: $0.14 ; 95 \% \mathrm{Cl}$ : $0.03-0.75 ; P$-value $=0.022$ ) retained statistical significance among floor samples, whereas the presence of chickens (OR: 4.73; 95\% Cl: 1.40-15.91; $P$-value $=0.012$ ) and table material (OR: 0.15; 95\% Cl: 0.03-0.82; $P$-value $=0.028$ ) retained statistical significance among table samples, adjusted for all other covariates.

Avian mitochondrial markers CytB and ND5. Fifty-nine percent $(62 / 104)$ of floor samples and $67.3 \%(70 / 104)$ of table samples were positive for CytB, whereas $83.7 \%(87 / 104)$ floor samples and $77.9 \%$ (81/104) table samples were positive for ND5. A greater number of wood tables (84.0\% [63/75]) were positive for the avian ND5 marker, in comparison to nonwood tables $(62.1 \%$ [18/29]) $(P$-value $=0.016)$. The median quantity of the avian markers CytB (wood: $4.31 \log _{10} \mathrm{GCN} / \mu \mathrm{L}$; non-wood $3.37 \log _{10} \mathrm{GCN} / \mu \mathrm{L} ; P$-value $=0.038$ ) and ND5 (wood: $4.24 \log _{10} \mathrm{GCN} / \mu \mathrm{L}$; non-wood $3.43 \log _{10} \mathrm{GCN} / \mu \mathrm{L}$ $P$-value $=0.010$ ) was statistically different between wood and non-wood tables.

Of significance, wood tables in comparison to table surfaces made of material other than wood had higher odds of being classified as "high" tertile, for both the ND5 (OR: 3.11; 95\% Cl: 1.38-7.02; $P$-value $=0.006)$ and CytB markers (OR: 2.53; 95\% Cl: 1.12-5.68; $P$-value $=0.025)$. Within the final adjusted model for ND5, the presence of chickens in the household (OR: 3.33; 95\% Cl: 1.33-8.30; $P$-value $=0.10)$ and treatment of drinking water (OR: 3.62; 95\% Cl: 1.44-9.12; $P$ value $=0.006$ ) retained statistical significance among floor samples, whereas table material (OR: 0.27; 95\% Cl: $0.10-0.76 ; P$-value $=0.013$ ) and treatment of drinking water (OR: $2.46 ; 95 \% \mathrm{Cl}: 1.04-5.79 ; P$-value $=0.040$ ) retained statistical significance among table samples. Within the final multivariable model for $C y t B$, the OR of a floor sample being classified in the "high" tertile in comparison with the "middle" and "low" tertiles was 0.94 (95\% Cl: 0.89-0.99; $P$-value = $0.015)$, for every additional age of the primary caregiver. The effect of treatment of drinking water (OR: $2.67 ; 95 \% \mathrm{Cl}$ : $1.12-6.39 ; P$-value $=0.027$ ) also retained statistical significance among table samples.

Dog fecal marker Bactcan. A total of $27.9 \%$ (29/104) of floors and $11.5 \%(12 / 104)$ of tables $(P$-value $=0.003)$ were positive for the dog fecal marker Bactcan. More unfinished floors (35.2\% [19/54]) were positive than finished floors (20.0\% [10/50]). Wood tables were $12.0 \%$ (9/75) positive and non-wood tables $10.3 \%$ (3/29). Once the quantities of Bactcan were categorized, $38.5 \%$ of floor samples and $11.5 \%$ of table were classified as having "high," $8.7 \%$ of floors and $5.8 \%$ of tables were classified as "medium," and $52.9 \%$ of floors and $82.7 \%$ of tables were classified as having "low" quantities of Bactcan marker $(P$-value $<0.001)$. From the univariate models, the odds of having a "high" Bactcan floor sample in comparison to a "medium" or "low" Bactcan floor sample were reduced by $70 \%(95 \% \mathrm{Cl}: 0.11-0.82 ; P$-value $=0.020)$ among households with wall materials other than cement in comparison to those households with cement walls. No statistically significant associations were found between the categories of Bactcan and household infrastructure and socioeconomic characteristics.

Human fecal markers Bachum and HF183-Taqman. A total of $17.3 \%(18 / 104)$ of floors and $3.9 \%(4 / 104)$ of tables were positive for the human fecal marker Bachum. Eight of the floors were unfinished and 10 were finished. All four tables positive for the human fecal marker were made of wood. When categorized, $31.7 \%$ of floors and $8.7 \%$ of tables had a high Bachum burden, whereas $68.3 \%$ of floors and $91.3 \%$ of tables 

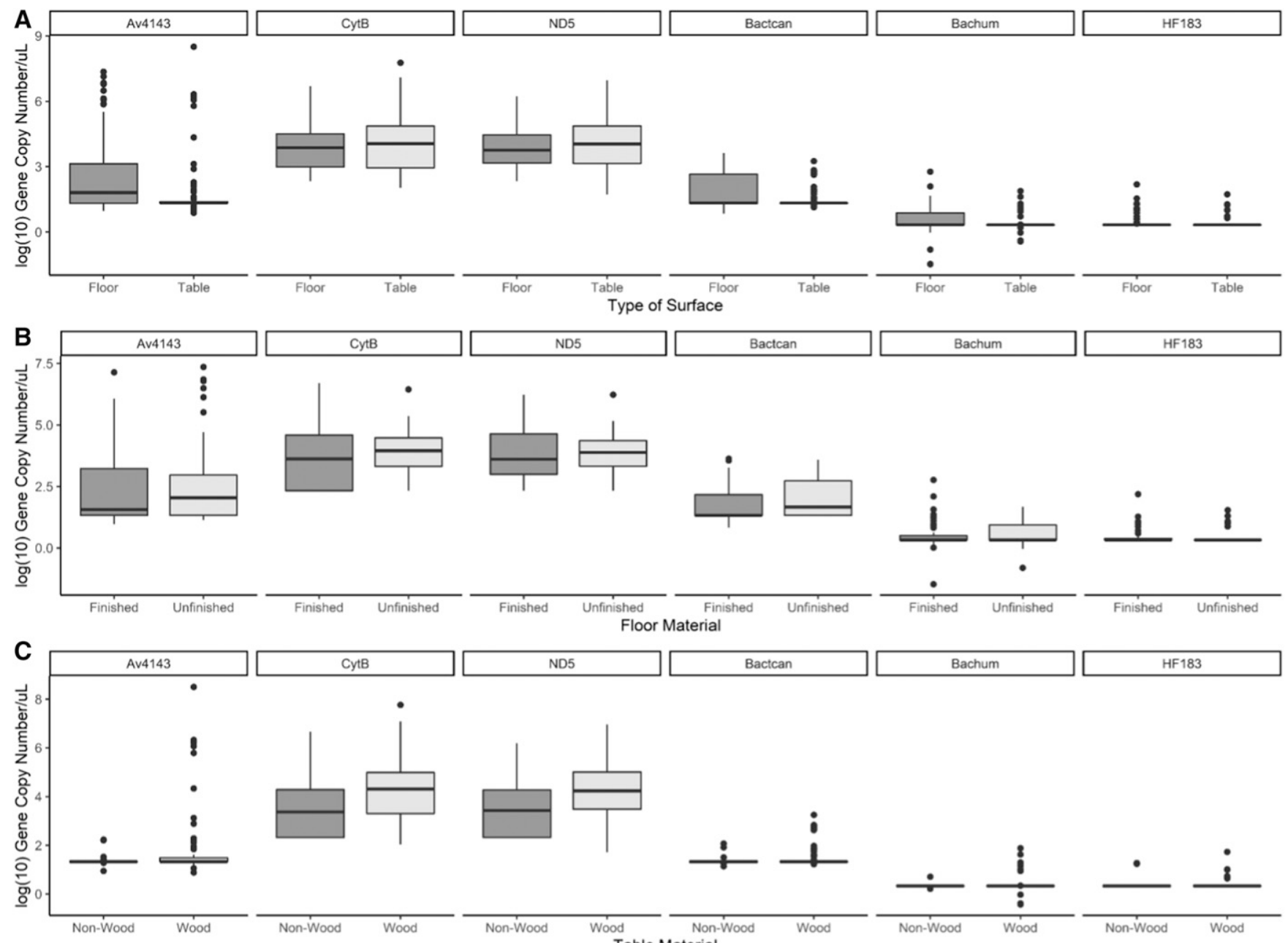

Floor Materia
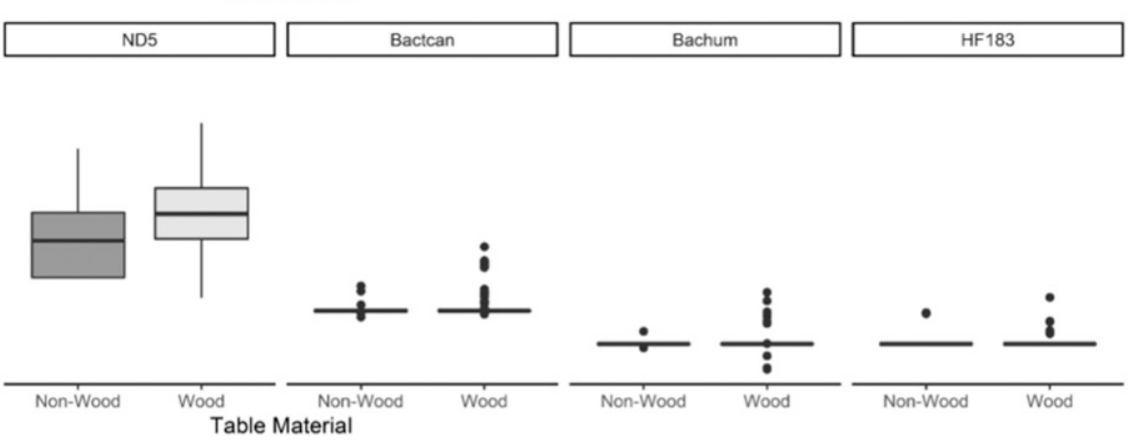

FIGURE 1. Quantitative burden of microbial source tracking markers in (A) floor and table samples, (B) unfinished and finished floors, and (C) wood and non-wood samples from households in three communities of lquitos, Peru. ${ }^{*}$ unfinished = dirt floor surface samples; finished = cement, tile, wood, or plastic floor surface samples; non-wood = tile, plastic, paper, or cloth table surface samples. Higher burden of animal feces in comparison to human feces in table and wood surfaces. Statistically significant higher burden of avian mitochondrial DNA and dog feces in floors in comparison to tables.

had a low Bachum burden $(P$-value $<0.001)$. Only one table and one floor were positive for the HF183-Taqman marker, which consisted of one finished floor and one wood table. In the final adjusted model, the odds of a table sample being classified in the "high" tertile in comparison with the "middle" and "low" tertiles were 0.81 (95\% Cl: 0.68-0.97; $P$-value = 0.020 ), for every additional age of the primary caregiver. Finally, in floor variables scored with the human Bachum fecal marker, the odds being classified in the "high" tertile in comparison with the "medium" and "low" tertiles were 13.81 (95\% $\mathrm{Cl}$ : 1.72-110.92; $P$-value $=0.014$ ) among samples from households with unimproved water sources in comparison to households with improved water sources, holding all other variables constant. This point estimate should be interpreted with caution, given the small sample size associated with the model and associated high Cls.

Microbial source tracking markers in toy samples. A total of 55 toys were given to a child and collected within 24 hours. Avian fecal contamination exceeded microbial contamination from other sources: ND5 (avian exposure) $41.8 \%$ (23/55), CytB (avian exposure) 20.0\% (11/55), Av4143 (avian feces) $7.3 \%$ (4/55), and BacHum (human feces) 3.6\% (2/55). All toy samples were negative for HF183-Taqman (human feces), Bactcan (canine feces), LA35 (avian feces), and Pig2Bac (swine feces). Of the 40 households with positive toys, $15.0 \%(6 / 40)$ had negative corresponding table or floor samples.

Association between Campylobacter spp. detection and MST markers. A total of $60.6 \%$ (63/104) floor surface samples (43 unfinished and 20 finished; $P$-value $<0.001)$ and $18.3 \%(19 / 104)$ table surface samples (16 wood and 3 nonwood; $P$-value $=0.193$ ) were positive for Campylobacter spp. (16S gene; $P$-value $<0.001)$. Among floor samples, the OR of detecting Campylobacter spp. was 29.34 (95\% Cl: 7.61-113.17; $P$-value < 0.001) among samples positive for Av4143, adjusting for the presence of chickens and floor material. Among table samples, the OR of detecting Campylobacter spp. was 29.49 (95\% Cl: 6.71-129.55; $P$-value $<0.001$ ) among samples positive for Av4143, adjusting for the presence of chickens and table material. Similarly, there was a higher odds of detecting Campylobacterspp. in floor and table samples if samples were positive for CytB (floors: OR: 12.61; 
TABLE 3

Female head of household, infrastructure, and hygiene characteristics by floor and table materials from households located in three communities of lquitos, Peru

\begin{tabular}{|c|c|c|c|c|c|c|}
\hline \multirow[b]{2}{*}{ Covariate } & \multicolumn{3}{|c|}{ Floors } & \multicolumn{3}{|c|}{ Tables } \\
\hline & Finished $(n=50)$ & Unfinished $(n=54)$ & $P$-value & Wood $(n=75)$ & Non-wood $(n=29)$ & $P$-value \\
\hline \multicolumn{7}{|l|}{ Female head of household characteristics } \\
\hline Maternal age (years), mean (95\% Cl) & $28.2(26.1-30.4)$ & $30.5(27.6-33.3)$ & 0.217 & $29.0(26.9-31.1)$ & $30.4(26.8-34.0)$ & 0.515 \\
\hline Maternal education, mean $(95 \% \mathrm{Cl})$ & $8.7(7.9-9.5)$ & $7.75(7.04-8.46)$ & 0.085 & $8.0(7.3-8.6)$ & $8.9(8.0-9.8)$ & 0.103 \\
\hline $\begin{array}{l}\text { Age of first pregnancy (years), mean } \\
(95 \% \mathrm{Cl})\end{array}$ & $18.6(17.2-20.1)$ & $17.6(16.8-18.4)$ & 0.212 & $17.7(16.8-18.7)$ & $19.0(17.4-20.5)$ & 0.175 \\
\hline $\begin{array}{l}\text { Monthly income (Peruvian sol), mean } \\
(95 \% \mathrm{Cl})\end{array}$ & $374.0(333.5-414.5)$ & $314.1(275.9-352.2)$ & 0.033 & $323.5(296.3-350.6)$ & $398.3(327.8-468.8)$ & 0.016 \\
\hline \multicolumn{7}{|l|}{ Household infrastructure characteristics } \\
\hline $\begin{array}{l}\text { Number of people sleeping in } \\
\text { household, mean (SD) }\end{array}$ & $6.4(5.7-7.0)$ & $5.8(5.1-6.5)$ & 0.209 & $6.2(5.6-6.8)$ & $5.6(4.8-6.3)$ & 0.216 \\
\hline $\begin{array}{l}\text { Length of household tenancy (years), } \\
\%(n / N)\end{array}$ & - & - & 0.228 & & & 0.739 \\
\hline Less than $1(n=12)$ & $6.0(3 / 50)$ & $16.7(9 / 54)$ & - & $10.7(8 / 75)$ & $6.9(2 / 29)$ & - \\
\hline Between 1 and $5(n=28)$ & $32.0(16 / 50)$ & $22.2(12 / 54)$ & - & $29.3(22 / 75)$ & $20.7(6 / 29)$ & - \\
\hline Between 5 and $10(n=22)$ & $16.0(8 / 50)$ & $25.9(14 / 54)$ & - & $22.7(17 / 75)$ & $20.7(6 / 29)$ & - \\
\hline Between 10 and $20(n=14)$ & $14.0(7 / 50)$ & $13.0(7 / 54)$ & - & $12(9 / 75)$ & $17.2(5 / 29)$ & _- \\
\hline More than $20(n=28)$ & $32.0(16 / 50)$ & $22.2(12 / 54)$ & - & $25.3(19 / 75)$ & $34.5(10 / 29)$ & - \\
\hline Wall type \% $(n / N)$ & - & - & - & - & - & - \\
\hline Cement & $36(18 / 50)$ & $11.1(6 / 54)$ & 0.003 & $16(12 / 75)$ & $48.3(14 / 29)$ & $<0.001$ \\
\hline Other & $64(32 / 50)$ & $88.9(48 / 54)$ & & $84(63 / 75)$ & $51.7(15 / 29)$ & \\
\hline \multicolumn{7}{|l|}{ Hygiene characteristics \% $(n / N)$} \\
\hline Household treats drinking water & $62.0(31 / 50)$ & $61.1(33 / 54)$ & 0.926 & $62.7(47 / 75)$ & $58.6(17 / 29)$ & 0.704 \\
\hline Water Source & - & - & & & & \\
\hline Improved & $90.0(45 / 50)$ & $88.9(48 / 54)$ & 0.854 & $86.7(65 / 75)$ & $96.6(28 / 29)$ & 0.142 \\
\hline Unimproved & $10.0(5 / 50)$ & $11.1(6 / 54)$ & & $13.3(10 / 75)$ & $3.4(1 / 29)$ & \\
\hline Sanitation facility & - & - & & & & \\
\hline Improved & $60.0(30 / 50)$ & $38.9(21 / 54)$ & 0.031 & $42.7(32 / 75)$ & $65.5(19 / 29)$ & 0.037 \\
\hline Unimproved & $40.0(20 / 50)$ & $61.1(33 / 54)$ & & $57.3(43 / 75)$ & $34.5(10 / 29)$ & \\
\hline Chickens in household $(n=50)$ & $54(27 / 50)$ & $42.6(23 / 54)$ & 0.245 & $49.3(37 / 75)$ & $51.7(15 / 29)$ & 0.827 \\
\hline
\end{tabular}

95\% Cl: 4.11-38.68; $P$-value < 0.001; tables: OR: 10.72; 95\% Cl: $1.36-84.75 ; P$-value $=0.025)$ and among floor samples if they were positive for ND5 (OR: 10.25 ; $95 \% \mathrm{Cl}: 2.43-43.32 ; P$ value $=0.002)$. The OR of detecting Campylobacter spp. in floor samples was 25.25 among samples positive for the human Bachum (95\% Cl: 2.94-216.81; $P$-value $=0.003)$. $\mathrm{A}$ multivariable model adjusting for the presence of all markers, as well as the presence of chickens in the household and the floor material, showed that the odds of having a Campylobacter spp.-positive sample increased by 11.71 (95\% Cl: 2.59-53.09; $P$-value $=0.001$ ) among floor samples positive for the Av4143 marker, and decreased by 0.11 (95\% Cl: 0.03-0.39; $P$-value $=0.001$ ) if floors were finished in comparison to unfinished floors. All other markers showed no association with the odds of detecting Campylobacter spp. in a floor sample in models adjusted for all markers, suggesting that identifiable exposures rather than universal communitywide contamination drove household levels of risk for campylobacteriosis. Similarly, among table samples, the OR of having a Campylobacter spp.-positive sample was 21.74 (95\% Cl: 4.62-101.38; $P$-value < 0.001) among samples positive for Av4143 in comparison to negative samples, adjusting for all other markers, presence of chickens, and table material. No other covariate was associated with the presence of Campylobacter spp. from a table surface sample.

\section{DISCUSSION}

In three peri-urban communities of lquitos, Peru, the prevalence of animal fecal material, most notably avian-derived contamination, exceeded that of human fecal material in structured sampling of household surfaces. Specifically, $76.9 \%$ household surfaces were positive for any fecal MST marker, $75.0 \%$ of households were positive for animal feces, and $20.2 \%$ were positive for human feces. Detection of avian markers was associated with the material of the surface of the table sampled, presence of chickens in the household, maternal age, and length of property tenance, whereas detection of human markers was associated with unimproved water source. This study further demonstrated that detection of viable Campylobacter spp. was associated with detection of avian marker Av4143, even after adjustment for the presence of chickens and the presence of other markers. Overall, this study strongly suggests that animal sources are important to fecal contamination in households in a tropical low-resource community.

Chickens are highly ubiquitous animals in communities in the Peruvian Amazon, given that poultry is the main source of animal protein besides fish, and are commonly raised as an alternative source of income or as pets. Chickens are seldom corralled, and there is no physical barrier that prohibits their entrance to the living or cooking spaces.

The odds of detecting high quantities of $A v 4143, C y t B$, and $N D 5$ were strongly associated with the presence of chickens in the household, corroborating the utility of these MST markers in this particular setting. In addition, having a positive Av4143 floor or table sample had a strong association with Campylobacter spp.-positive surface samples. Although chickens are considered a risk factor for Campylobacter spp. infections, we do not advocate discouraging the practice of 


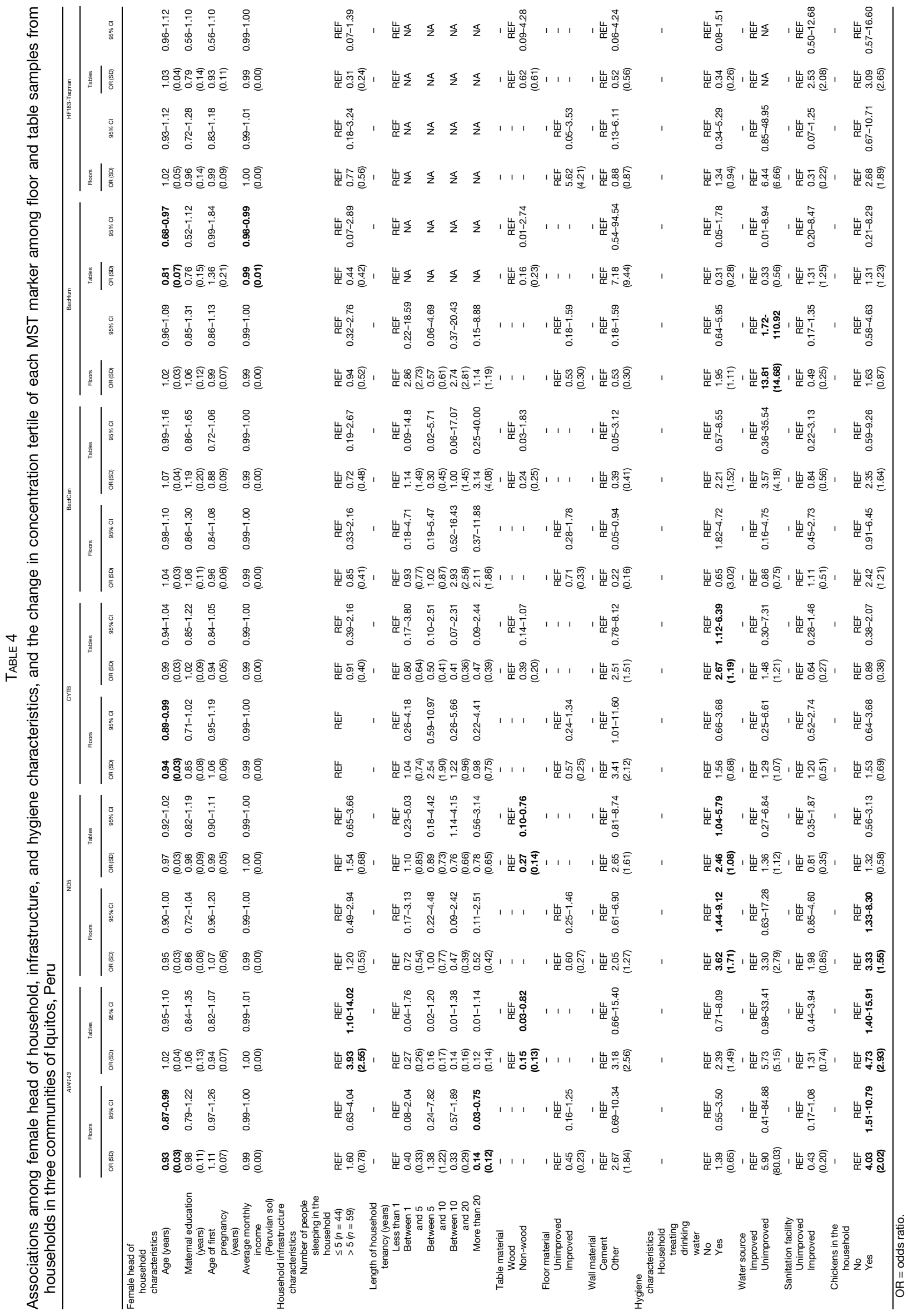


chicken rearing. On the contrary, chickens and eggs are an important nutritional source of protein and iron. However, these data suggest the potential importance of mitigation strategies to reduce exposure to avian fecal material, and future studies will be needed to identify strategies that are effective but that do not have unintended consequence for economic stability of households, animal health, or human health. In fact, studies in Peru and Ethiopia present evidence that suggests corralling chickens might increase the risk of Campylobacter spp. transmission and infection, potentially by affecting the ecology of Campylobacter spp. in the host or by increasing the degree of animal crowding and, therefore, overall concentration of fecal burden in a single location within the household. ${ }^{42,43}$ Results from this study indicate that a higher burden of avian exposure, as measured by CytB and ND5, is associated with wood tables than non-wood tables. This is one potential risk factor amenable to intervention that could be tested directly in future trials, given cooking tables made or covered of materials such as plastic or tiles are more frequently cleaned than non-wood tables (personal observation). Recent randomized clinical trials that aim to reduce the burden of enteric disease using water, hygiene, and sanitation practices have not been unsuccessful. ${ }^{44-46}$ Although reasons for these are multifaceted, some studies have already suggested the need to incorporate household infrastructure improvements to reduce the burden of fecal contamination. Among many, these include building cement floors, improved water distribution systems, and improved waste management systems. A recent call to for an "integrative management of animals, wash, sanitation, and hygiene" highlights the need for transformative Water, Sanitation, and Hygiene interventions. ${ }^{47}$ This study provides data to support the importance of addressing animal fecal waste within household environments and to introduce a One Health approach to water sanitation and hygiene research.

We found few samples positive for the human fecal markers Bachum and HF183-Taqman. However, among samples that were positive for Bachum, the odds of having high human fecal contamination were highly increased if the household had unimproved water. Although human MST markers have been most frequently used in resource-poor environments, to our knowledge, they have not been applied to household surface samples. ${ }^{20,21,48,49}$ Pig2Bac and LA35 markers were not detected in any surface sample. The lack of positive samples to Pig2Bac is not surprising, given that few households own and raise pigs. The absence of positive LA35 markers is attributed to the particularly low sensitivity of this marker. ${ }^{29}$

Limitations of this study include the fact that the performance of MST markers is setting specific and requires a previous validation step. In addition, new human MST markers that have higher degrees of sensitivity and specificities are required. Given the emerging microbiome research, the development of markers taking into consideration age-specific features of fecal microbiota, age-specific Bifidobacterium, and other fastidious members of the flora could significantly enhance our understanding of the source of human fecal contamination. It is important to note that the lack of positive surface samples to certain MST outcomes may have prevented the precise estimation of associations. However, results show a consistent positive association between the presence of Campylobacter and avian MST markers, despite the stated sample size limitation.
Future studies should include water samples along with surface samples as a further method of comparison, as well as samples from toilet areas and human hands, as hand-tomouth ingestion of avian fecal material has been shown previously. ${ }^{50}$ Comparing the burden of MST markers in these additional sampling environments will help elucidate the degree of animal and human fecal waste among potential transmission pathways that exist in these environments. Finally, the isolation of viable Campylobacter from surface samples would have strengthened our findings.

Human and avian fecal markers as well as avian exposure markers were detected among the 55 toys sampled. The high degree of positivity to avian and human markers suggests that toys serve as a relevant sentinel of animal fecal exposure and that both avian and human feces are frequently in contact with household members.

\section{CONCLUSION}

Avian fecal material is highly prevalent in floor and cooking spaces of households located in peri-urban, low-resource tropical communities. There is a need to include animal fecal waste in interventions that target water, hygiene, and sanitation aiming to reduce the burden of enteric disease and environmental enteropathy. However, care should be taken to do this in a way that recognizes the key food security role chickens and eggs play in pediatric and adult populations living in resource-poor settings and should be used to shape, rather than discourage, domestic animal husbandry practices. This study adds to mounting evidence for the need to treat the domestic environment as a single entity using a One Health strategy, and to support simple interventions to decrease exposure to avian fecal material and potentially pathogenic bacteria such as the use of easy-to-clean plastic surfaces on cooking tables.

Received July 6, 2020. Accepted for publication September 7, 2020.

Published online November 2, 2020.

Note: Supplemental tables appear at www.ajtmh.org.

Acknowledgments: We wish to express our heartfelt thanks to the community members of Santa Clara, Santo Tomas, and La Union for allowing us to enter their homes. We thank Victoria Lopez Manuyama, Judith Chavez Rengifo, Zoila Huiñapi Nolorbe, Erika Linares Robalino, Silvia Rengifo Pinedo, Ruth Rodriguez Aquituari, Marvin Yalta Navarro, and Fiorella Zegarra Paredes for their contribution to sample collection and processing.

Financial support: F. S. was supported by FONDECYT-CONCYTEC (grant contract number 246-2015-FONDECYT), the NIH Fogarty Global Health Fellows Consortium composed of Johns Hopkins University, the University of North Carolina, Morehouse University, and Tulane University (grant no. D43TW009340), and partial support by the Fogarty International Center NIH Training grant no. D43 TW001140. N. P. was supported by a gift from the GRACE Communications Foundation, NIEHS grant R01ES026973, and NIAID grants R21Al139784 and R43Al141265. M. N. K. was supported by the University of Virginia departments of Infectious Diseases and Internal Medicine and the Bill \& Melinda Gates Foundation (OPP1066146). M. F. D. was supported by the National Institutes of Health (K01OD019918 to M. F. D.) and the Canine Health Foundation (02241).

Authors' addresses: Francesca Schiaffino, Faculty of Veterinary Medicine, Universidad Peruana Cayetano Heredia, Lima, Peru, E-mail: francesca.schiaffino@upch.pe. Dixner Rengifo Trigoso, Maribel Paredes Olortegui, Wagner V. Shapiama Lopez, and F. Garcia Bardales, Asociacion Benefica Prisma, Iquitos, Peru, E-mails: dixrentri@ gmail.com,mparedeso@prisma.org.pe,wagnervalentino.wvsl@ 
gmail.com, and pafrgarcia@gmail.com. Josh M. Colston and Pablo Penataro Yori, Division of Infectious Diseases and International Health, University of Virginia School of Medicine, Charlottesville, VA, E-mails: josh.colston@virginia.edu and pyori@virginia.edu. Nora Pisanic and Meghan F. Davis, Department of Environmental Health and Engineering, Johns Hopkins Bloomberg School of Public Health, Baltimore, MD, E-mails: npisani1@jhu.edu and mdavis65@jhu.edu. Margaret N. Kosek, Department of Molecular and Comparative Pathobiology, Johns Hopkins Bloomberg School of Medicine, Baltimore, MD, E-mail: mkosek@virginia.edu.

This is an open-access article distributed under the terms of the Creative Commons Attribution (CC-BY) License, which permits unrestricted use, distribution, and reproduction in any medium, provided the original author and source are credited.

\section{REFERENCES}

1. Julian TR, 2016. Environmental transmission of diarrheal pathogens in low and middle income countries. Environ Sci Process Impacts 18: 944-955.

2. Huda TMN, Schmidt WP, Pickering AJ, Unicomb L, Mahmud ZH, Luby SP, Biran A, 2019. Effect of neighborhood sanitation coverage on fecal contamination of the household environment in rural Bangladesh. Am J Trop Med Hyg 100: 717-726.

3. Julian TR, MacDonald LH, Guo Y, Marks SJ, Kosek M, Yori PP, Pinedo SR, Schwab KJ, 2013. Fecal indicator bacteria contamination of fomites and household demand for surface disinfection products: a case study from Peru. Am J Trop Med Hyg 89: 869-872.

4. Exum NG, Olortegui MP, Yori PP, Davis MF, Heaney CD, Kosek M, Schwab KJ, 2016. Floors and toilets: association of floors and sanitation practices with fecal contamination in Peruvian Amazon Peri-Urban households. Environ Sci Technol 50: 7373-7381.

5. Mattioli MC, Pickering AJ, Gilsdorf RJ, Davis J, Boehm AB, 2013. Hands and water as vectors of diarrheal pathogens in Bagamoyo, Tanzania. Environ Sci Technol 47: 355-363.

6. Exum NG, Kosek MN, Davis MF, Schwab KJ, 2017. Surface sampling collection and culture methods for Escherichia coli in household environments with high fecal contamination. Int $J$ Environ Res Public Health 14: 947.

7. Pickering AJ, Julian TR, Marks SJ, Mattioli MC, Boehm AB, Schwab KJ, Davis J, 2012. Fecal contamination and diarrheal pathogens on surfaces and in soils among Tanzanian households with and without improved sanitation. Environ $\mathrm{Sci}$ Technol 46: 5736-5743.

8. Bukenya GB, Nwokolo N, 1991. Compound hygiene, presence of standpipe and the risk of childhood diarrhoea in an urban settlement of Papua New Guinea. Int J Epidemiol 20: 534-539.

9. Zambrano LD, Levy K, Menezes NP, Freeman MC, 2014. Human diarrhea infections associated with domestic animal husbandry: a systematic review and meta-analysis. Trans $R$ Soc Trop Med Hyg 108: 313-325.

10. Headey D, Nguyen P, Kim S, Rawat R, Ruel M, Menon P, 2017. Is exposure to animal feces harmful to child nutrition and health outcomes? A multicountry observational analysis. Am J Trop Med Hyg 96: 961-969.

11. Ercumen A et al., 2017. Animal feces contribute to domestic fecal contamination: evidence from $E$. coli measured in water, hands, food, flies, and soil in Bangladesh. Environ Sci Technol 51: 8725-8734.

12. Fuhrmeister ER et al., 2019. Predictors of enteric pathogens in the domestic environment from human and animal sources in rural Bangladesh. Environ Sci Technol 53: 10023-10033.

13. Byappanahalli MN, Roll BM, Fujioka RS, 2012. Evidence for occurrence, persistence, and growth potential of Escherichia coli and enterococci in Hawaii's soil environments. Microbes Environ 27: 164-170.

14. Fujioka R, Sian-Denton C, Borja M, Castro J, Morphew K, 1998. Soil: the environmental source of Escherichia coli and Enterococci in Guam's streams. J Appl Microbiol 85 (Suppl 1): 83S-89S.

15. Boehm AB, Van De Werfhorst LC, Griffith JF, Holden PA, Jay JA, Shanks OC, Wang D, Weisberg SB, 2013. Performance of forty- one microbial source tracking methods: a twenty-seven lab evaluation study. Water Res 47: 6812-6828.

16. Field KG, Samadpour M, 2007. Fecal source tracking, the indicator paradigm, and managing water quality. Water Res 41: 3517-3538.

17. Jenkins MW, Tiwari S, Lorente M, Gichaba CM, Wuertz S, 2009. Identifying human and livestock sources of fecal contamination in Kenya with host-specific Bacteroidales assays. Water Res 43: 4956-4966.

18. Schriewer A, Odagiri M, Wuertz S, Misra PR, Panigrahi $P$, Clasen $\mathrm{T}$, Jenkins MW, 2015. Human and animal fecal contamination of community water sources, stored drinking water and hands in rural India measured with validated microbial source tracking assays. Am J Trop Med Hyg 93: 509-516.

19. Ahmed W, Yusuf R, Hasan I, Goonetilleke A, Gardner T, 2010. Quantitative PCR assay of sewage-associated bacteroides markers to assess sewage pollution in an urban lake in Dhaka, Bangladesh. Can J Microbiol 56: 838-845.

20. Odagiri M, Schriewer A, Hanley K, Wuertz S, Misra PR, Panigrahi $P$, Jenkins MW, 2015. Validation of Bacteroidales quantitative PCR assays targeting human and animal fecal contamination in the public and domestic domains in India. Sci Total Environ 502: 462-470.

21. Nshimyimana JP, Cruz MC, Thompson RJ, Wuertz S, 2017. Bacteroidales markers for microbial source tracking in Southeast Asia. Water Res 118: 239-248.

22. Harris AR, Pickering AJ, Harris M, Doza S, Islam MS, Unicomb L, Luby S, Davis J, Boehm AB, 2016. Ruminants contribute fecal contamination to the urban household environment in dhaka, Bangladesh. Environ Sci Technol 50: 4642-4649.

23. Boehm AB et al., 2016. Occurrence of host-associated fecal markers on child hands, household soil, and drinking water in rural Bangladeshi households. Environ Sci Technol Lett 3: 393-398.

24. Holcomb DA, Knee J, Sumner T, Adriano Z, de Bruijn E, Nala R, Cumming O, Brown J, Stewart JR, 2020. Human fecal contamination of water, soil, and surfaces in households sharing poor-quality sanitation facilities in Maputo, Mozambique. Int $J$ Hyg Environ Health 226: 113496.

25. Amour $\mathrm{C}$ et al.; Etiology, Risk Factors, and Interactions of Enteric Infections and Malnutrition and the Consequences for Child Health and Development Project (MAL-ED) Network Investigators, 2016. Epidemiology and impact of Campylobacter infection in children in 8 low-resource settings: results from the MAL-ED study. Clin Infect Dis 63: 1171-1179.

26. Lee G, Pan W, Penataro Yori P, Paredes Olortegui M, Tilley D, Gregory M, Oberhelman R, Burga R, Chavez CB, Kosek M, 2013. Symptomatic and asymptomatic Campylobacter infections associated with reduced growth in Peruvian children. PLoS Negl Trop Dis 7: e2036.

27. Lee G, Paredes Olortegui M, Penataro Yori P, Black RE, Caulfield L, Banda Chavez C, Hall E, Pan WK, Meza R, Kosek M, 2014. Effects of Shigella-, Campylobacter- and ETEC-associated diarrhea on childhood growth. Pediatr Infect Dis J 33: 1004-1009.

28. Carron M et al., 2018. Campylobacter, a zoonotic pathogen of global importance: prevalence and risk factors in the fastevolving chicken meat system of Nairobi, Kenya. PLoS Negl Trop Dis 12: e0006658.

29. Schiaffino F, Pisanic N, Colston JM, Rengifo D, Olortegui MP, Shapiama V, Yori PP, Heaney CD, Davis MF, Kosek MN, 2020. Validation of microbial source tracking markers for the attribution of fecal contamination in indoor-household environments of the Peruvian Amazon. Sci Total Environ 743: 140531.

30. Yori PP et al., 2014. Santa Clara de Nanay: the MAL-ED cohort in Peru. Clin Infect Dis 59 (Suppl 4): S310-S316.

31. Davis MF, Baron P, Price LB, Williams DL, Jeyaseelan $S$, Hambleton IR, Diette GB, Breysse PN, McCormack MC, 2012. Dry collection and culture methods for recovery of methicillinsusceptible and methicillin-resistant Staphylococcus aureus strains from indoor home environments. Appl Environ Microbiol 78: 2474-2476.

32. Vujcic J, Ram PK, Hussain F, Unicomb L, Gope PS, Abedin J, Mahmud ZH, Islam MS, Luby SP, 2014. Toys and toilets: crosssectional study using children's toys to evaluate environmental 
faecal contamination in rural Bangladeshi households with different sanitation facilities and practices. Trop Med Int Health 19: 528-536.

33. Weidhaas JL, Macbeth TW, Olsen RL, Sadowsky MJ, Norat D, Harwood VJ, 2010. Identification of a Brevibacterium marker gene specific to poultry litter and development of a quantitative PCR assay. J Appl Microbiol 109: 334-347.

34. Ohad S, Ben-Dor S, Prilusky J, Kravitz V, Dassa B, Chalifa-Caspi $\mathrm{V}$, Kashi Y, Rorman E, 2016. The development of a novel qPCR assay-set for identifying fecal contamination originating from domestic fowls and waterfowl in Israel. Front Microbiol 7: 145.

35. Zhuang FF, Li H, Zhou XY, Zhu YG, Su JQ, 2017. Quantitative detection of fecal contamination with domestic poultry feces in environments in China. AMB Express 7: 80.

36. Kildare BJ, Leutenegger CM, McSwain BS, Bambic DG, Rajal VB, Wuertz S, 2007. 16S rRNA-based assays for quantitative detection of universal, human-, cow-, and dog-specific fecal Bacteroidales: a Bayesian approach. Water Res 41: 3701-3715.

37. Pisanic N, Nadimpalli M, Rinsky JL, Stewart J, Wing S, Love DC, Hall D, Heaney CD, 2015. Pig-2-Bac as a biomarker of occupational exposure to pigs and livestock-associated Staphylococcus aureus among industrial hog operation workers. Environ Res 143: 93-97.

38. Platts-Mills JA et al., 2014. Detection of Campylobacter in stool and determination of significance by culture, enzyme immunoassay, and PCR in developing countries. J Clin Microbiol 52: 1074-1080.

39. Botteldoorn N, Van Coillie E, Piessens V, Rasschaert G, Debruyne L, Heyndrickx M, Herman L, Messens W, 2008. Quantification of Campylobacter spp. in chicken carcass rinse by real-time PCR. J Appl Microbiol 105: 1909-1918.

40. Florey L, Taylor C, 2016. Using Household Survey Data to Explore the Effects of Improved Housing Conditions on Malaria Infection in Children in Sub-Saharan Africa. DHS Analytical Studies No. 61. Rockville, MD: ICF International.

41. WHO, 2019. Progress on Household Drinking Water, Sanitation and Hygiene 2000-2017. Special Focus on Inequalities. New
York, NY: United Nations Children's Fund (UNICEF) World Health Organization.

42. Oberhelman RA, Gilman RH, Sheen P, Cordova J, Zimic M, Cabrera L, Meza R, Perez J, 2006. An intervention-control study of corralling of free ranging chickens to control Campylobacter infections among children in a Peruvian periurban shantytown. Am J Trop Med Hyg 74: 1054-1059.

43. Headey D, Hirvonen K, 2016. Is exposure to poultry harmful to child nutrition? An observational analysis for rural Ethiopia. PLoS One 11: e0160590.

44. Luby SP et al., 2018. Effects of water quality, sanitation, handwashing, and nutritional interventions on diarrhoea and child growth in rural Bangladesh: a cluster randomised controlled trial. Lancet Glob Health 6: e302-e315.

45. Null $\mathrm{C}$ et al., 2018. Effects of water quality, sanitation, handwashing, and nutritional interventions on diarrhoea and child growth in rural Kenya: a cluster-randomised controlled trial. Lancet Glob Health 6: e316-e329.

46. Pickering AJ et al., 2019. The WASH Benefits and SHINE trials: interpretation of WASH intervention effects on linear growth and diarrhoea. Lancet Glob Health 7: e1139-e1146.

47. Prendergast AJ et al., 2019. Putting the "A" into WASH: a call for integrated management of water, animals, sanitation, and hygiene. Lancet Planet Health 3: e336-e337.

48. Odagiri $\mathrm{M}$ et al., 2016. Human fecal and pathogen exposure pathways in rural Indian villages and the effect of increased latrine coverage. Water Res 100: 232-244.

49. Malla B, Ghaju Shrestha R, Tandukar S, Bhandari D, Inoue D, Sei K, Tanaka Y, Sherchand JB, Haramoto E, 2018. Validation of host-specific Bacteroidales quantitative PCR assays and their application to microbial source tracking of drinking water sources in the Kathmandu Valley, Nepal. J Appl Microbiol 125: 609-619.

50. Marquis GS, Ventura G, Gilman RH, Porras E, Miranda E, Carbajal L, Pentafiel M, 1990. Fecal contamination of shanty town toddlers in households with non-corraled poultry, Lima, Peru. Am J Public Health 80: 146-149. 